\title{
System Architectures for Sensor-Based Dynamic Remaining Shelf-life Prediction
}

\author{
Åse Jevinger, Malmö University, Malmö, Sweden \\ Paul Davidsson, Malmö University, Malmö, Sweden
}

\begin{abstract}
Different storage and handling conditions in cold supply chains often cause variations in the remaining shelf life of perishable foods. In particular, the actual shelf life may differ from the expiration date printed on the primary package. Based on temperature sensors placed on or close to the food products, a remaining shelf-life prediction (RSLP) service can be developed, which estimates the remaining shelf life of individual products, in real-time. This type of service may lead to decreased food waste and is used for discovering supply chain inefficiencies and ensuring food quality. Depending on the system architecture, different service qualities can be obtained in terms of usability, accuracy, security, etc. This article presents a novel approach for how to identify and select the most suitable system architectures for RSLP services. The approach is illustrated by ranking different architectures for a RSLP service directed towards the supply chain managers. As a proof of concept, some of the most highly ranked architectures have been implemented and tested in food cold supply chains.
\end{abstract}

\section{KEYWORDS}

AHP, Cold Supply Chain, Implementation, Information System Architecture, Perishable Food, Remaining Shelf-life Prediction, Sensor-based System, Temperature Sensor

\section{INTRODUCTION}

Studies show that temperature in food supply chains (FSCs) often differ from the ones recommended by the producer (Hafliðason et al., 2012; Likar and Jevšnik, 2006). This might lead to shorter shelf life for perishables, such as dairy products, fresh meat and fish, and that the expiry dates printed on the primary packages no longer represent good estimates of the actual shelf life. On the other hand, food that has been well treated can often be safely consumed after the printed expiry date has passed. It has been estimated that one third of all food produced globally for human consumption is lost or wasted and that more than $40 \%$ of this food loss occur at retail and consumer levels, in industrialized countries (Gustavsson et al., 2011). With information about the actual shelf life of the products, this food waste can be reduced. One way to get an estimate closer to the actual shelf life than the printed expiry date is to use product condition monitoring. That is, to continuously measure relevant conditions of the products, such as temperature, and use these measurements to estimate the product shelf life.

Among environmental parameters in FSCs, temperature has the most significant influence on the quality of perishable food products (Jedermann et al., 2009). As a consequence, temperature abuse and fluctuations are the main reasons for product returns, food waste and financial losses (Raab et al., 2008). Scheer (2006) reports that mismanaged temperature during perishable FSC distribution can cause up to $35 \%$ in product loss. Moreover, Göransson et al. (2018a) have found significant differences in estimated product shelf life when comparing different FSCs for the same product types 
and temperature requirements, in field tests. This means that the corresponding differences between actual shelf life and the shelf life printed on the packages, also varied. By using local sensors to measure the temperature in FSCs, the actual quality of the food products can be estimated. These sensors must be present throughout the transport chain, and the closer to the products they are placed, the more accurate estimations can be made. Based on the estimated quality, the remaining shelf life can be dynamically updated in real time, enabling product owners, actors within supply chain management and final consumers, to make more informed decisions about how to handle the food and when to discard it. Furthermore, a service providing a remaining shelf-life prediction (RSLP) based on temperature sensors might be colligated with other services such as tracking and tracing, product information (for instance origin and handling instructions), carbon dioxide labeling, and dynamic pricing (Bartels et al., 2010).

An RSLP service can be implemented in many different ways, using different system architectures. For instance, the remaining shelf life can be calculated on the product or by remote servers (in the cloud), and the temperature can be measured by a sensor attached to the product or by fixed close-by device. These different options need to be evaluated before an informed decision about the system architecture can be taken. To the best of our knowledge, there are no previous studies on how this can be done in a systematic manner for this type of service. In his paper, we present and apply a novel approach to identify and evaluate the most suitable system architecture for an RSLP service for perishable food. We illustrate the approach by ranking all architectures satisfying the requirements of two particular user groups: the supply chain managers and product owners. As a proof of concept, we have also implemented and tested some of the most highly ranked architectures in a cold FSC. In summary, the aims of this paper is to:

- Present and apply a novel approach to identify and evaluate the most suitable system architecture for an RSLP service,

- Present experiences gained from implementing and testing some of the most suitable RSLP architectures in a cold FSC,

- Prove that this type of service can be implemented using the technology available today.

The remainder of this paper is structured as follows. The next two sections describe previous studies related to product condition monitoring, and the methodology applied in this paper. Thereafter, we present the results in terms of a new model for representing the system architectures, a filtering process leading to a set of architectures subject for evaluation, the final ranking of these architectures, and the experiences gained from FSC implementation and tests (as proof of concept). The paper ends with a short discussion and summary of conclusions.

\section{PRODUCT CONDITION MONITORING: STATE-OF-THE-ART}

Products accompanied by a higher level of intelligence than only possessing an associated ID (e.g. the simplest form of RFID), are often referred to as "smart" or "intelligent" (Meyer et al., 2009). For instance, these products may be capable of communicating with their environments, storing data, processing data, and even making autonomous decisions. Depending on the level of intelligence, such products may be capable of monitoring the local conditions during transportation (López et al., 2011), and maybe also of calculating the remaining shelf life. The intelligence related to a product may or may not be located on the product itself (Meyer et al., 2009). However, for the RSLP service, local intelligence, for instance implemented on the product, a pallet or in the vehicle, able to perform condition monitoring is required.

Within the area of communicative packaging, potential means to monitor the condition of packaged contents by enhancing the intelligence of the package itself have been investigated (Heising 
et al., 2014). The solutions consist of a combination of packaging technologies and communicative signals (e.g. changing colors, diagrams, displays) (Dobon et al., 2011). Communicative packaging is seen as a specific type of smart packaging, which uses different technologies to add extra features to packaging (e.g. information about remaining shelf life, identification about the type/origin of the product and protection against counterfeiting). A life cycle assessment study on the use of a flexible best-before-date communicative device (FBBD, with a temperature logger and a display) on packaging consumer units, shows that the use of FBBD devices decreases environmental burdens associated to the production, packaging and delivery to the point of sale, thanks to reduction in food losses (Dobon et al., 2011). Other case studies show potential benefits of radio-frequency identification (RFID) based cold-chain monitoring in increased sales due to reduced out-of-stock, reduction in inventory due to lower safety stock, improvement of visibility and transparency in the supply chain etc. (Jol et al., 2006). Benefits from using TTIs (Sahin et al., 2007; Bhushan and Gummaraju, 2002) and wireless sensor networks (Qi et al., 2014) have also been investigated. In particular, Bijwaard et al. (2011) have studied the communication links in a wireless sensor network capable of monitoring environmental conditions, calculating the remaining product shelf life, and transmit this data to a back-end server. In recent years, FSC monitoring based on the Internet of Things (IoT) umbrella concept have emerged (Wang and Yue, 2017). For instance, data collected by means of IoT can be represented in virtual objects, to allow for monitoring, controlling, planning and optimizing remotely and in real time (Verdouw et al., 2016).

An alternative to enhancing the package of a product itself is to place the local intelligence, including temperature sensors, on a higher level, such as on containers or in vehicles (Ruiz-Garcia et al., 2007; Jedermann et al., 2014). For instance, Sciortino et al. (2016) have developed a smart logistics unit (SLU) mounted on pallets, that measures humidity, temperature and different types of gases, and communicates these measurements, together with GPS information, to a server.

Placing intelligence on different levels in a transport system results in different processing, information and communication requirements as well as enables different service qualities and functionalities. Depending on the purpose of local condition monitoring and shelf-life estimations, different solutions based on different system architectures might thereby be preferred - in particular by different target user groups. As shown above, several previous studies have investigated how to monitor food products. Some of them have also used the collected sensor data to calculate product shelf life. Although different system architectures can be distinguished in the previous studies, none of the studies addresses the problem of how to identify the different architecture alternatives and how to determine which one to choose. This paper aims at filling this research gap.

\section{METHODOLOGY}

The research methodology applied in this paper is based on design science (DS) research in information systems (IS) (Hevner et al., 2004). This discipline is directed towards creating applicable solutions to problems, by developing artifacts, at the intersection of IT and organizations (Peffers et al., 2007). According to this terminology, the artifact developed within our study is a method for identifying and evaluating appropriate system architectures. Hevner et al. (2004) present a framework for understanding, executing, and evaluating information systems (IS) research. The framework includes the environment, which defines the problem space and the business needs, and the knowledge base, which provides the raw materials to the IS research (theories, frameworks, etc.). The business needs and the knowledge base form the input to the IS research process. The principal business needs for our research have been described in the introduction. The knowledge base is composed of conceptual modelling and existing expertise concerning field tests and questionnaires.

In order to determine necessary requirements for the RSLP service and the corresponding system architecture, we used the results from a previous study, in which we interviewed 11 different FSC actors (Jevinger et al., 2014). Additionally, we conducted literature studies to identify relevant criteria. 
In order to value the identified criteria, we then asked five actors within the FSC, four different perishable food producers and one supply chain manager, to fill in a questionnaire. These actors were selected to reflect the user groups in focus in this paper. The questionnaire asked each respondent to set a value (between 1 and 10) to each of the identified criteria (see Appendix 1).

As mentioned above, we have developed a novel approach to identify and evaluate the most suitable system architecture for an RSLP service. The information collected from the interviews, questionnaire and literature review was used to specify the target user group requirements, in this approach. We also developed a system architecture representation model, as part of the approach. The model characterizes architectures based on where (e.g., directly at the product or at a remote server) the different activities involved in the RSLP service (e.g., reading and storing sensor data, and computing the RSLP) are performed. The model is used to identify the set of all possible system architectures. Thereafter, the candidates that do not meet the target user group requirements are removed from the set. Moreover, we use conceptual filtering principles to remove architecture candidates that do not seem reasonable from a practical perspective. The remaining system architectures are then evaluated using an Analytical Hierarchical Process (AHP), which is a method for analyzing complex decision problems with multiple criteria (Saaty, 1980). The criteria are used in pairwise comparisons of the considered alternatives and should reflect all important aspects of each of the alternatives (Saaty, 1987).

We illustrate the novel approach to identify the most suitable system architectures, by applying it to two particular user groups, the supply chain managers and product owners. Some of the most highly ranked system architectures were then implemented and tested in real cold FSCs, involving food producers, logistics service providers, wholesalers, distributors, and retailers. Some of the system requirements from the previous study also affected the implementation of this architecture (Jevinger et al., 2014). Thereby, these served as requirements during the implementing phase, as well.

In this study, the RSLP is based on temperature measurements. Thus, sensors are needed to record the local conditions according to some time interval. Theoretically, the service could have been based on measuring the current bacteria level only. However, given the current status of sensor technology, direct measurement of the current bacteria level in a product is not a viable approach.

\section{RESULTS}

In order to be able to compare different system architectures of an RSLP service, they must first be identified and characterized. We have developed a new system architecture representation model, that characterizes the architectures based on where the different activities involved in the service are performed. The model was developed by first specifying all activities (A) that must be performed, as well as the different locations (L) where each of these activities could be performed. By combining the activities with the locations, we got the set of all potential architectures (i.e., the Cartesian product $L^{|A|}$ ).

The set of potential architectures was then reduced by removing unfeasible and obviously impractical architectures. From the remaining set of architectures, the most suitable ones, with respect to the functional and quality requirements of each target user group, should be found. Ideally, every identified architecture should be evaluated and compared. However, due to the amount of work this process would require, the least suitable ones were filtered out in two steps. In the first filtering step, we applied target user group requirements specifying where the RSLP should be presented. For instance, some user groups might want to reach the RSLP via a mobile terminal (e.g. terminal workers) whereas others might want to read it on the product itself (e.g. consumers). In this paper, we only focus on the architectures satisfying the requirements of the supply chain managers and product owners, which are both assumed to require the RSLP displayed in a remote device, for instance a stationary computer running an ERP system. In the second filtering step, two conceptual filtering principles derived from general requirements on the architecture were applied to distinguish the most promising candidates.

Finally, AHP analysis was performed on the resulting set of architectures, prioritizing the most suitable ones according to a set of quality-based criteria. As mentioned above, the criteria used should 
reflect all the important aspects of each of the considered alternatives. In our case, we identified a part of these criteria by analyzing the service requirements expressed by FSC actors. Interviews from a previous study identified following system requirements (Jevinger et al., 2014):

1. The solution must be affordable.

2. The solution should be seamlessly integrated in the FSC.

3. The generated information should be reported directly into the Enterprise Resource Planning (ERP) systems.

4. Temperature must be measured close to the products.

5. Generated information must be protected from unauthorized access (e.g. from competitors)

6. Generated information must be protected from data tampering.

System requirements no. 2 and 3, affect the implementation of an architecture. Requirements no. 4-6 influence the choice of architecture. These requirements were therefore used, in combination with quality attributes gathered from literature (Offutt, 2002; Barbacci et al., 1995; O'Brien et al., 2007; Larsson, 2004; Clements, 2001), to determine the criteria for the AHP. In order to value the identified criteria, we collected data from the questionnaire. The results from this questionnaire served as input to the AHP.

Requirement 1 naturally also influences the final choice of architecture. However, since costs are dependent on current prices and technological achievements, cost aspects are not included in the AHP. Instead, the architectures are prioritized from a functional and quality perspective. Cost aspects are taken into consideration after the AHP.

Figure 1 illustrates the different steps in the novel approach used for identifying possible system architectures for RSLP services and evaluating the most suitable ones.

In the following subsections we first present and apply the system architecture representation model (step 1 in Figure 1). We then describe the target user group requirements as well as the conceptual filtering principles used (step 2 and 3 in Figure 1). This is followed by the application of the Analytical Hierarchical Process to evaluate the remaining system architectures (step 4 in Figure 1). The section ends with a proof of concept, presenting the experiences gained from our FSC implementations and tests of some of the most highly ranked architectures resulting from the preceding application of steps 1-4.

\section{System Architecture Representation Model}

The RSLP service requires the execution of four main activities: $\left(a_{1}\right)$ the local temperature of the product have to be measured, $\left(a_{2}\right)$ the recorded temperature data must be stored, $\left(a_{3}\right)$ the RSLP must be calculated based on the stored temperature data, and $\left(\mathrm{a}_{4}\right)$ the result must be presented to the user of the service. These activities $\left(A=\left\{a_{1}, a_{2}, a_{3}, a_{4}\right\}\right)$ can be performed at different locations: at product level $(\mathrm{P})$, on a fixed local device $(\mathrm{N})$, on a mobile local device $(\mathrm{M})$, in a remote device $(\mathrm{R})$ or in the

Figure 1. Approach to identify and evaluate the most suitable system architectures for RSLP services

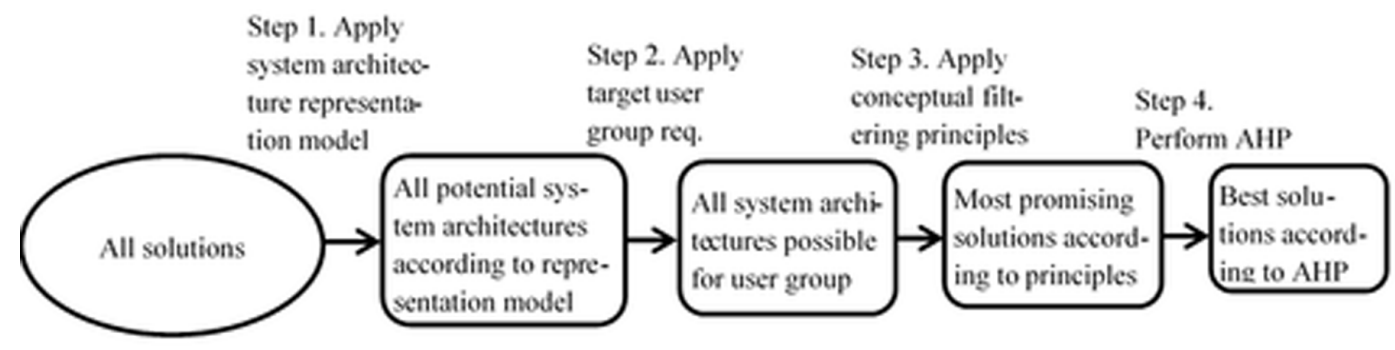


cloud (C), i.e. $\mathrm{L}=\{\mathrm{P}, \mathrm{N}, \mathrm{M}, \mathrm{R}, \mathrm{C}\}$. Below, a more detailed explanation of the different locations is given. Figure 2 illustrates the corresponding possible communication links between these locations. In this study, we assume that both $\mathrm{R}$ and $\mathrm{M}$ are connected with the cloud (when there is coverage) and that $\mathrm{N}$ is available whenever needed in accordance with the requirements of the architecture.

The entities in set $\mathrm{L}$ can be explained as follows:

P: Entity at product level, i.e., attached to the primary package. This entity follows the product throughout the transport.

$\mathbf{N}$ : Local device near the products, located for instance on the secondary package, pallet, or inside a vehicle, terminal or refrigerator. The $\mathrm{N}$ used by the service on behalf of a product may change during transport.

M: Mobile local device within communication range of the product or local device. This could be a smartphone used by consumers or local supply chain personnel, e.g. truck drivers and terminal workers. M may communicate directly with $\mathrm{N}$ and $\mathrm{P}$.

R: Remote device, for instance a stationary computer running an ERP system. $\mathrm{R}$ is unable to communicate directly with $\mathrm{N}$ and $\mathrm{P}$.

C: Cloud entities, for instance network servers, accessible from any device with Internet access.

Given the number of activities and the number of possible locations above, the set of potential architectures, $L^{\mid A \backslash}$, consists of 625 elements $(5 * 5 * 5 * 5=625)$. However, some of these correspond to unfeasible or obviously impractical solutions. Measuring the local conditions in $\mathrm{C}$ or $\mathrm{R}$ is impossible, and to do it on $\mathrm{M}$ is impractical since $\mathrm{M}$ may be moved around. Also, to store the sensor data on $\mathrm{M}$ or $\mathrm{N}$ is impractical since the service should not be dependent on $\mathrm{M}$ being present (receiving sensor data via WPAN/RFID) and since storing sensor data on $\mathrm{N}$ would require a data transfer whenever $\mathrm{N}$ changes and that might not be possible (or desirable for security reasons). Finally, to present the expiration date in $\mathrm{C}$ is not meaningful. To sum up, in reality the activities may only be executed in the following locations in the system:

Figure 2. Possible communication links between different parts of the service system, (W)WAN: (Wireless) Wide Area Network, WLAN: Wireless Local Area Network WPAN: Wireless Personal Area Network.

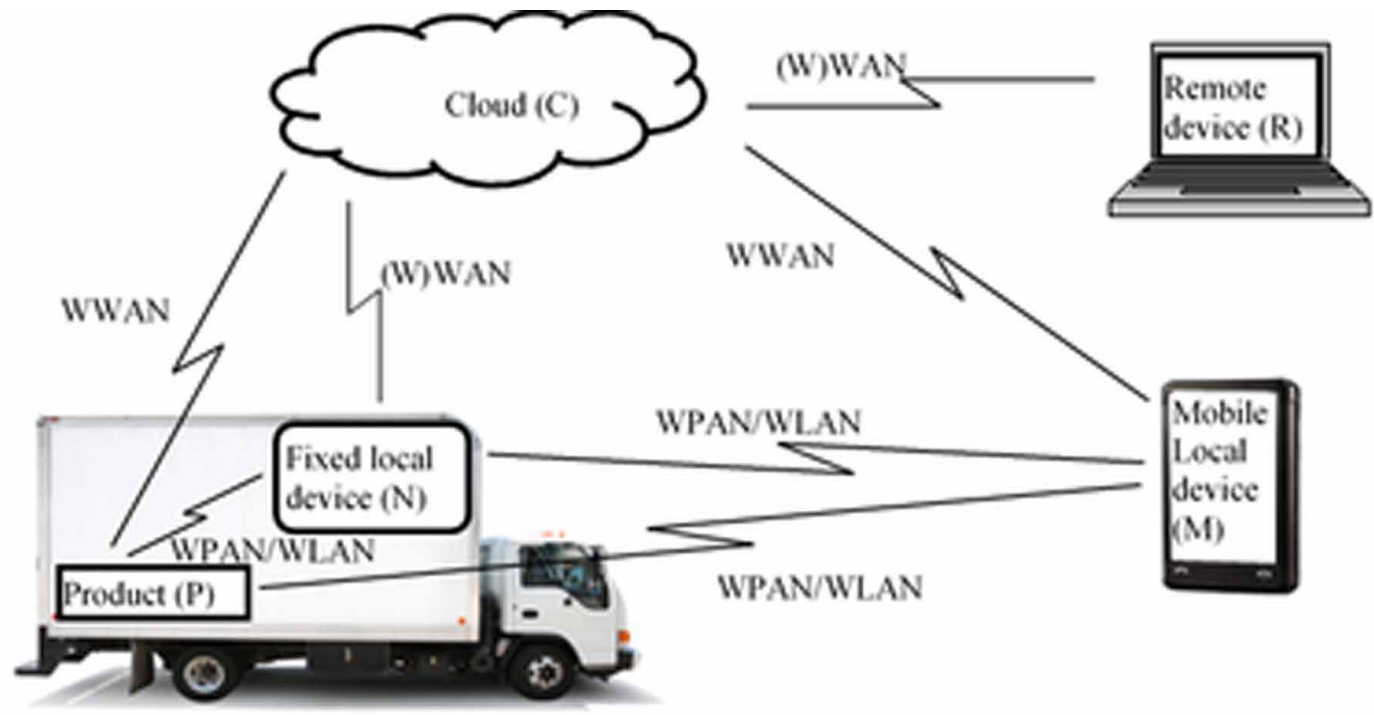


1. Measurement of local conditions: $\mathrm{P}$ or $\mathrm{N}$

2. Sensor data storage: $\mathrm{P}, \mathrm{C}$ or $\mathrm{R}$

3. RSLP calculation: $\mathrm{P}, \mathrm{N}, \mathrm{M}, \mathrm{R}$ or $\mathrm{C}$

4. RSLP presentation: $\mathrm{P}, \mathrm{N}, \mathrm{M}$, or R

The resulting RSLPs are assumed to be stored either where they are calculated or where they are to be presented in the system (or not at all). The RSLP calculation is based on current and historical sensor data and this sensor data might, on the other hand, be stored somewhere apart from where it is collected or used. The reason for storing this data in a third place might be to avoid overloading the product level or to allow for the fixed local device to be changed during transport. Depending on where the sensor data is stored, a higher level of intelligence might be required by the involved entities, as well as additional communication.

The options listed above results in 120 different alternative architectures $(2 * 5 * 4 * 3=120)$. We have investigated each one of them with respect to individual characteristics, usefulness for different target user groups, communication paths and required capabilities (according to Jevinger et al., 2011).

The architectures can be grouped based on where measurement, storage and calculation are located. All architectures with the same locations of these activities only differ in where the RSLP is presented, and they may therefore be combined to provide several means of accessing the RSLP. For instance, a solution showing the RSLP in both the R and M might be required (e.g. to satisfy requirements from drivers and terminal workers as well as centralized supply chain managers), based on architectures with the same locations of the other service activities (measurement, storage and calculation). In this study, we show an approach to how to prioritize the most suitable solutions for one of the target user groups; however, this approach can be used to prioritize the solutions for other user groups as well. If a combination is required as described, the topmost architectures with the same locations of measurement, storage and calculation should be selected from the resulting prioritized lists of architectures.

\section{Target User Group Requirements and Conceptual Filtering Principles}

Different user groups, e.g. consumers, terminal workers, truck drivers, supply chain managers, product owners, etc., require different alternatives for how the RSLP should be accessed. This paper focuses on the supply chain managers and product owners, which we assume only have one requirement on how they want to read the RSLP, namely in a remote device (R). For instance, they might want the RSLP service integrated with their ERP system. Ideally, all architectures fulfilling this requirement should be evaluated with respect to the functional and quality preferences of the supply chain managers/ product owners. This would mean evaluating 48 architectures. For other user groups identified, the set of architectures to evaluate has been shown to be 138 and performing an AHP on all of these would require a huge amount of work. Moreover, many of the architectures that are possible from a theoretical perspective reflect solutions that do not seem reasonable from a practical perspective, such as calculating the RSLP in M and showing the result on P. Therefore, we have developed two principles for filtering out the least promising architectures, from the perspective of the supply chain managers/product owners. The list of principles may have to be extended for other user groups, though the principles below should be applied for those as well.

Conceptual Filtering Principle 1: If sensor data is stored on $C / R$ and the RSLP is presented on $R$, the calculation should be co-located with the storage or the presentation. The purpose of this principle is to prioritize the simplest solutions that do not involve sending information back and forth, in particular from the central level, to the local level, and back again. For instance, architectures storing sensor data in R, using P/N for calculation, and showing the resulting RSLP in R, are removed. This principle filters out the following architectures (Measurement-Storage-Calculation-Presentation): P-C-P-R, N-C-P-R, P-C-N-R, N-C-N-R, P-C-M-R, N-C-M-R, P-R-M-R, N-R-M-R, P-R-C-R, N-RC-R, P-R-N-R, N-R-N-R, P-R-P-R, N-R-P-R. 
Conceptual Filtering Principle 2: Architectures showing the RSLP on R shall not use $\mathrm{M}$ for calculation. The supply chain managers/product owners should not be dependent on $\mathrm{M}$ for receiving the RSLP since M is mobile and is therefore not guaranteed to always be present. This principle filters out the following architectures: P-P-M-R, N-P-M-R.

As a result, 14 architecture candidates remain that have not been rejected by the principles. Thus, the following AHP will be based on these.

\section{Analytical Hierarchical Process}

AHP is an approach to evaluating a number of different alternatives with respect to several criteria, and based on this, select the best option. Pairwise comparisons are used to develop overall priorities reflecting the importance of each criterion, relative to the goal of the selection problem. The performance of each alternative on each criterion must also be determined, and together with the priorities of the criteria, a ranking of the alternatives can be produced. In this paper, we use AHP to rank the remaining architecture candidates. AHP has previously been successfully used for evaluating architecture candidates (Svahnberg et al., 2003; Davidsson et al., 2006). As a first step, the set of criteria must be identified. Based on a large set of quality attributes found in literature (Offutt, 2002; Barbacci et al., 1995; O'Brien et al., 2007; Larsson, 2004; Clements, 2001), in combination with a number of RSLP service requirements from a previous study (Jevinger et al., 2014), we have identified the criteria listed in Table 1 as relevant for the RSLP service.

The second step of AHP involves prioritizing the criteria according the importance of each criterion, in relation to the goal of the selection problem. Each criterion is pairwise compared to all other criteria with respect to how desired they are to the service system. In our case, these comparisons were based on the questionnaires given to actors operating in different parts of the FSC (see methodology section). We only used consistent priorities, and after the pairwise comparisons, the priorities were normalized to sum up to one. The questionnaire responses can be found in Appendix 2 , and the resulting normalized priorities are listed in the first row of Table 2.

In the third step of AHP, the architecture candidates are compared with all other candidates, with respect to the criteria. These comparisons are based on pairwise assessments and as before, we used consistent priorities, normalized to sum up to one. The resulting sets are shown in Table 2, below first row.

In the fourth step, the relative importance of each criterion is combined with how well each architecture candidate satisfy the criteria, by multiplying the normalized priorities related to the architecture candidates with the normalized priorities of the criteria. The resulting products are thereafter summed for each architecture candidate and the values of these sums reflect the suitability of each candidate, in relation to the other candidates. The final values for each architecture candidate are presented in the last column of Table 2.

Table 2 shows that, with this approach, architecture N-R-R-R receives the highest AHP score for the supply chain managers/product owners, with respect to quality and functionality requirements. Architecture P-R-R-R is second best, whereas the two similar architectures P-P-P-R and P-P-R-R are regarded as third best. Even though the accuracy is considered as most important and measurements on $\mathrm{P}$ have a much higher accuracy than measurements on $\mathrm{N}$, architecture N-R-R-R still receives higher priority due to its other advantages, primarily in response time, security and availability. Had the accuracy been considered as even more important by the questionnaire respondents, the priorities of the two architectures might have been reversed.

Taking into consideration the costs of each solution as well naturally would change the AHP scores. For instance, architecture P-R-R-R most probably entails a higher cost than architecture N-P-R-R, since architecture P-R-R-R requires sensor capability on P whereas architecture N-P-R-R may be implemented using a simple read/write passive RFID tag on P. Implementing a high level of intelligence in product-level devices is usually relatively costly since the number of products is high in relation to the other potential locations, for instance vehicles. Thus, the approach presented 
Table 1. Criteria for AHP analysis

\begin{tabular}{|l|l|}
\hline \multicolumn{1}{|c|}{ Criteria } & \multicolumn{1}{c|}{ Meaning } \\
\hline $\begin{array}{l}\text { Response } \\
\text { time }\end{array}$ & $\begin{array}{l}\text { How long it takes to process a request (O'Brien et al., 2007). We define it as the time it takes from } \\
\text { asking for the RSLP, until it is available for the user. Situations in which the RSLP cannot be provided } \\
\text { at all are covered by Availability. }\end{array}$ \\
\hline Security & $\begin{array}{l}\text { We consider integrity and confidentiality as the most relevant aspects of security. Integrity guarantees } \\
\text { that information is not corrupted and confidentiality ensures that access to the information/service is } \\
\text { granted only to authorized subjects (O'Brien et al., 2007). In our context integrity corresponds to how } \\
\text { difficult it is to manipulate the system into producing no or erroneous RSLPs, and confidentiality to } \\
\text { how difficult it is for unauthorized users to access the RSLP. }\end{array}$ \\
\hline Availability & $\begin{array}{l}\text { The proportion of time a system or component is operational and accessible (O'Brien et al., 2007). In } \\
\text { our context, it is measured as the proportion of the time the service is operational during its lifetime. We } \\
\text { do not address physical damage of equipment. }\end{array}$ \\
\hline Modifiability & $\begin{array}{l}\text { The ability to make changes to a system quickly and cost-effectively (Clements et al., 2001). In our } \\
\text { context, it is a measure of how easy and cost-effectively the software (date estimation algorithms, } \\
\text { software functionality etc.) and the hardware components (tags, readers, displays etc.) of the service can } \\
\text { be exchanged. }\end{array}$ \\
\hline Scalability & $\begin{array}{l}\text { The ability of the system to function well when the system is changed in size or in volume in order to } \\
\text { meet users' needs (O'Brien et al., 2007). In our context, it concerns how well the service system is able } \\
\text { to handle a growing number of products or longer transport distances. }\end{array}$ \\
\hline Accuracy & $\begin{array}{l}\text { The precision of computations and control (Larsson, 2004). In our context, it concerns how well the } \\
\text { RSLP approximates the actual remaining shelf life, including the reliability of the sensor data. }\end{array}$ \\
\hline
\end{tabular}

Table 2. Priorities derived from questionnaires and comparisons between the architecture candidates with respect to the criteria as well as the final results of the AHP analysis

\begin{tabular}{|c|c|c|c|c|c|c|c|}
\hline & Response time & Security & Availability & Modifiability & Scalability & Accuracy & $\begin{array}{l}\text { AHP } \\
\text { results }\end{array}$ \\
\hline Priorities & 0,166 & 0,170 & 0,175 & 0,157 & 0,144 & 0,188 & \\
\hline P-P-P-R & 0,077 & 0,073 & 0,073 & 0,102 & 0,046 & 0,158 & 0,090 \\
\hline N-P-P-R & 0,038 & 0,024 & 0,024 & 0,051 & 0,046 & 0,018 & 0,033 \\
\hline P-P-N-R & 0,077 & 0,073 & 0,073 & 0,051 & 0,023 & 0,158 & 0,079 \\
\hline N-P-N-R & 0,038 & 0,024 & 0,024 & 0,034 & 0,023 & 0,018 & 0,027 \\
\hline P-P-C-R & 0,051 & 0,036 & 0,036 & 0,102 & 0,046 & 0,158 & 0,073 \\
\hline P-C-C-R & 0,051 & 0,073 & 0,036 & 0,102 & 0,091 & 0,079 & 0,071 \\
\hline N-P-C-R & 0,044 & 0,018 & 0,018 & 0,051 & 0,046 & 0,018 & 0,031 \\
\hline N-C-C-R & 0,115 & 0,073 & 0,146 & $\mathbf{0 , 0 5 1}$ & 0,136 & $\mathbf{0 , 0 2 0}$ & 0,088 \\
\hline P-C-R-R & 0,051 & 0,073 & 0,036 & 0,102 & 0,091 & 0,079 & 0,071 \\
\hline N-C-R-R & 0,115 & 0,073 & 0,146 & 0,051 & 0,136 & 0,020 & 0,088 \\
\hline P-P-R-R & 0,077 & 0,073 & 0,073 & 0,102 & 0,046 & 0,158 & 0,090 \\
\hline N-P-R-R & 0,038 & 0,024 & 0,024 & 0,051 & 0,046 & 0,018 & 0,033 \\
\hline P-R-R-R & $\mathbf{0 , 0 7 7}$ & 0,146 & 0,073 & 0,102 & 0,091 & 0,079 & 0,094 \\
\hline N-R-R-R & 0,153 & 0,218 & 0,218 & 0,051 & 0,136 & 0,020 & 0,132 \\
\hline
\end{tabular}


in this paper should primarily be used for prioritizing the architectures from a functional and quality perspective and based on these results, in combination with the costs of each architecture candidate, the most suitable solution can finally be selected.

\section{Proof of Concept}

According to Table 2, the following architectures received the highest AHP scores: P-P-P-R, P-PN-R, P-P-C-R, P-C-C-R, N-C-C-R, P-C-R-R, N-C-R-R, P-P-R-R, P-R-R-R, N-R-R-R. However, as mentioned above, costs have not yet been considered. After having searched the market for available sensors and corresponding readers, we concluded that temperature sensors using Bluetooth Low Energy (BLE) technology would incur the lowest costs. For instance, RFID readers both cover a smaller area and are more expensive. The choice of using BLE caused some restrictions on the architectures, though. Of the architectures with highest priorities, P-P-P-R, P-P-N-R, P-P-C-R, P-PR-R, P-C-R-R and N-C-R-R (written in italics in Table 2) were not included in the cold FSC tests, for the following reasons:

- Letting the product level be responsible for both measuring the temperature and storing the data, requires a sensor with logging capabilities. However, we were unable to find BLE temperature sensors with logging capabilities on the market (without additional development costs included in the prize). Therefore, architectures P-P-P-R, P-P-N-R, P-P-C-R, P-P-R-R were excluded.

- As can be seen, the architectures P-C-R-R and P-C-C-R receive the same AHP score in Table 2. Thus, from a functional and quality perspective, these are equally well suited. The same goes for architectures N-C-R-R and N-C-C-R. For practical reasons, we chose only to implement P-C-C-R and N-C-C-R.

In summary, the cold FSC tests included the architectures P-C-C-R, N-C-C-R, P-R-R-R, N-R$\mathrm{R}-\mathrm{R}$ (written in boldface in Table 2).

The implementation included Bluetooth Low Energy temperature sensors (nRF51822, Nordic Semiconductor) measuring food product temperatures, smartphones (Sony Xperia) acting as gateways, a relational database storing all temperature data and a web server with PHP support, which calculated and distributed all results. The gateways were responsible for collecting temperature sensor data and transmitting it to the database. The data was then used by the web server to calculate the remaining shelf lives of the products involved, at any given time. The cold FSC tests included three different product types: fresh cod, smoked ham and cottage cheese. Different product-specific microbiological prediction models were implemented for each of the product types: Mataragas et al. (2006) for smoked ham, Dalgaard et al. (1997) for fresh cod and Østergaard et al. (2014) for cottage cheese. The selection of these models was based on previous microbiological studies within the project Dynahmat (Dynamic shelf life for minimised food waste (Dynahmat, 2016)), aiming at identifying models with the smallest bias and highest accuracy factor, in relation to measured bacteria levels. All FSC tests took place in the southern and middle parts of Sweden, and were carried out between 2014 and 2016, as part of the Dynahmat project. Both the database and the web server were deployed at an IBM platform called Bluemix. Figure 3 shows the system setup.

The communication between the gateway and the database was based on the HTTP POST protocol, over 3G. The information protocol was defined as part of the study, and it primarily included the following information: ID of the sensor, ID of the gateway, measured temperature, time of the measurement, and location of the gateway at the time of measurement. The last information entity was used for track and trace purposes, e.g. when temperature was below or above recommended limits.

The application formed by the database and the PHP scripts enables users to create representations of cold FSCs. The product type connected to a sensor is specified in the user interface, when the sensor is associated with a specific test. The product type determines which prediction model is used. Moreover, since the initial concentration of target bacteria has a strong influence on the shelf 


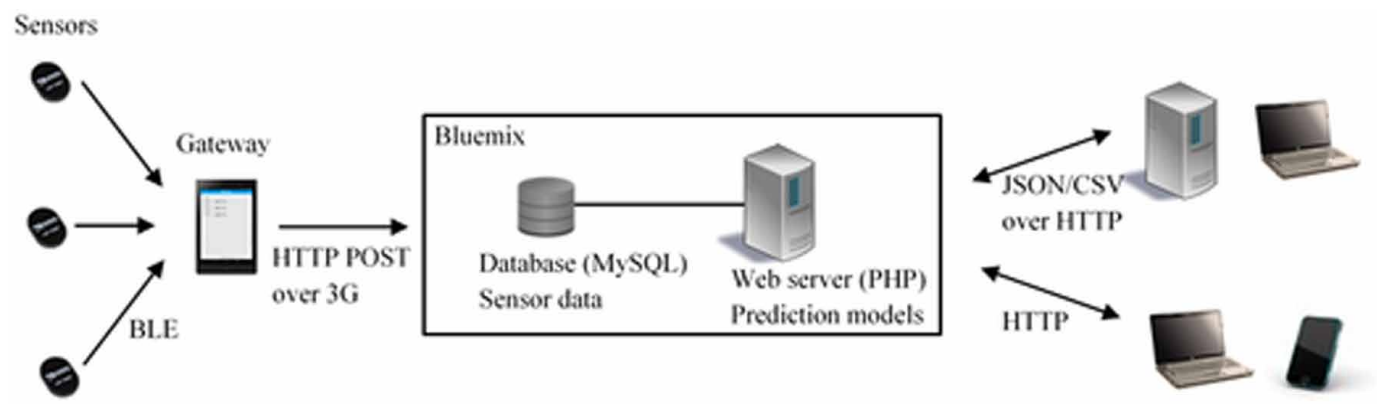

life of a product, this also has to be specified by the user. Since we have no information about future temperatures, two different versions of the RSLP are presented by the system: one based on the assumption that the product temperature is kept at the highest recommended temperature in the future, and one based on the assumption that the product temperature remains the same as the current temperature.

An important insight gained from the cold FSC tests was that the sensors acquired from retailers were rarely correctly calibrated, which caused discrepancies between the actual temperatures and the measured temperatures. Therefore, we investigated the calibration of each of the sensors in a thermal incubator. As a result, a linear equation ( $y=k x+m$, where $y$ represents the actual temperature and $x$ represents the measured temperature), had to be used to compensate for the error. The coefficient $(k)$ and the constant $(m)$ for each sensor were identified by the thermal incubator tests, and then inserted into the system via the user interface. We investigated the calibration of more than 100 sensors. However, for a commercial solution, this will most probably not be a viable approach.

According to the requirements on an RSLP service, the generated information should be reported directly into the ERP systems. To satisfy this requirement, the implemented system allows for data retrieval via an API. The default response format is JSON over HTTP but the data can also be fetched as CSV over HTTP (see Figure 3). The following data may be retrieved:

- Sensor data as received from the gateways (raw data)

- Actual temperature data (calibrated as described above)

- Prediction data

The prediction models used in the tests (for fresh cod, smoked ham and cottage cheese) share some similarities, which led to an implementation where the prediction models inherit from a generic prediction model. The shared functionality was defined in the generic model, whereas specifics and differences were handled by extending and overriding the generic model, in the specific model. However, in its present form, whenever new prediction models need to be added, the software must be altered. To overcome this problem, the microbiological models should be parameterized, so that when perishables with new prediction models are to be added to the system, these models can be specified via the user interface.

The implemented architectures, i.e. P-C-C-R, N-C-C-R, P-R-R-R and N-R-R-R, differ in sensor location as well data storage and calculation location. The differences in sensor location had no effect on our implementation, except for the number of sensors required. Moreover, as shown by Göransson et al. (2018b), the different sensor locations probably have little effect on the accuracy of the shelf-life predictions. The differences in data storage and calculation location primarily affected the security. When implemented in C, we had less control over the security, but had to rely on some of the services provided by Bluemix. 


\section{DISCUSSION AND CONCLUSION}

We have shown a novel approach to how to identify the most suitable system architectures of an RSLP service. As part of this approach, we have developed a system architecture representation model, which characterizes architectures based on where the different activities involved in the service are performed. The primary advantage with this model is that all possible architectures can be identified and evaluated. Thereafter, we have used user group specific requirements and two conceptual filtering principles to identify and remove architecture candidates that do not fulfill the general requirements on the final solution. The remaining set of candidates has been evaluated in an AHP based on a number of quality criteria, and the results are presented as a list of priorities of the candidates.

Furthermore, we have illustrated the novel approach to identify the most suitable system architectures, by applying it for two particular user groups, the supply chain managers and product owners. This work resulted in a list of the most highly ranked architectures. Some of these were then implemented and tested in cold FSCs. These tests proved that a working solution of this type of service can be implemented using the technology available today. The tests also validated that the method developed can be used to find a solution that can be implemented and used. The implemented solution is generic in the sense that it works for different types of sensors and food products.

These tests included software that provided shelf-life data based on product-specific prediction models. In our implementation, these models were incorporated with the system. However, depending on the business model behind the RSLP service, there may be other alternatives to this. For instance, the prediction models may reside within each FSC actor's own information system. This means that the actor would be responsible for producing the prediction data based on the sensor data, which is retrieved from the system via the APIs. Another alternative would be to let the FSC actors define and formalize the prediction models within each actor's domain, and then communicate them in a way that allows the system to use them. This would require a defined a messaging format for communications between the system and the actors. A conventional format for such tasks is XML.

Our implementation included a gateway communicating sensor data from the products, to the central database. One alternative to this would be to let the products communicate directly with the database, by using a low-power wide-area network protocol, e.g. NB-IoT or LoRa. Naturally, these communication technologies can also be used for any fixed local devices.

According to the system requirements identified in a previous study (Jevinger et al., 2014), the solution should preferable be integrated with the food producer systems used today. This means that the RSLP service should add as little extra work as possible, for instance, around sensor installations. There is no need for having sensors at every food product, though. Göransson et al. (2018b) emphasize the importance of continuous temperature monitoring along the entire cold food supply chains. However, they show that temperature sensors used for calculating the predicted shelf life of a product, can be placed relatively far from the product itself (e.g. on the secondary package or even on the pallet) without jeopardizing the reliability of the resulting shelf-life prediction.

Building up an infrastructure for an RSLP service may enable other services as well, e.g. temperature control, dynamic pricing, and track and trace. In particular, the actors participating in our studies have expressed specific interests for a common and reliable way of temperature measurements along the FSC. However, an RSLP service also raises many questions in terms of the business model. For a successful implementation, sensor data is required from the entire FSC, which means that many actors must be involved. Some of these gain more from the service than others. Who should pay for what investments, and who owns the generated data? These and many other associated questions need to be sorted out.

Future work involves implementation of the other system architectures that received high AHP scores, which would enable further validation. In particular, quantitative evaluation of different architectures in practical implementations would be useful. Moreover, further studies on how an RSLP service would affect the behaviors of consumers and supply chain actors are needed, as well as investigations related to the user interface design. 


\section{ACKNOWLEDGMENT}

Parts of the work presented in this paper has been conducted within the interdisciplinary project Dynahmat, funded by the TvärLivs programme managed by VINNOVA (Swedish Governmental Agency for Innovation Systems). 


\section{REFERENCES}

Barbacci, M., Longstaff, T. A., \& Weinstock, C. B. (1995). Quality Attributes. Carnegie Mellon University.

Bartels, P.V., Tromp, S.O., Rijgersberg, H., \& Kreft, F. (2010). Improvement of sustainability in the perishable food supply chain by using communicative packaging devices. In Towards Effective Food Chains: Models and Applications (pp. 275-291).

Bhushan, N., \& Gummaraju, K. (2002). A Petri net based simulation approach for evaluating benefits of time temperature indicator and wireless technologies in perishable goods retail management. In The Second International Conference on Simulation and Modeling in the Food and Bio-Industry (FOODSIM'2002).

Bijwaard, D. J., van Kleunen, W. A., Havinga, P. J., Kleiboer, L., \& Bijl, M. J. (2011). Industry: Using dynamic WSNs in smart logistics for fruits and pharmacy. In The 9th ACM Conference on Embedded Networked Sensor Systems.

Clements, P., Kazman, R., \& Klein, M. (2003). Evaluating software architectures. Beijing: Tsinghua University Press.

Dalgaard, P., Mejlholm, O., \& Huss, H. H. (1997). Application of an iterative approach for development of a microbial model predicting the shelf-life of packed fish. International Journal of Food Microbiology, 38(2), 169-179. doi:10.1016/S0168-1605(97)00101-3 PMID:9506282

Davidsson, P., Johansson, S., \& Svahnberg, M. (2006). Using the Analytic Hierarchy Process for Evaluating Multi-Agent System Architecture Candidates. In J. Müller \& F. Zambonelli (Eds.), Agent-Oriented Software Engineering VI (pp. 205-217). Berlin, Germany: Springer. doi:10.1007/11752660_16

Dobon, A., Cordero, P., Kreft, F., Østergaard, S. R., Robertsson, M., Smolander, M., \& Hortal, M. (2011). The sustainability of communicative packaging concepts in the food supply chain. A case study: Part 1. Life cycle assessment. The International Journal of Life Cycle Assessment, 16(2), 168-177. doi:10.1007/s11367-011-0257-y

Dynahmat. (2016). Report: Insights and learnings from the challenge-driven innovation project DynahMat. Retrieved from http://dynahmat.com

Göransson, M., Jevinger, A., \& Nilsson, J. (2018b). Shelf-life variations in pallet unit loads during perishable food supply chain distribution. Food Control, 84, 554-560. doi:10.1016/j.foodcont.2017.08.027

Göransson, M., Nilsson, F., \& Jevinger, ̊. (2018a). Temperature performance and food shelf-life accuracy in cold food supply chains-Insights from multiple field studies. Food Control, 86, 332-341. doi:10.1016/j. foodcont.2017.10.029

Gustavsson, J., Cederberg, C., Sonesson, U., van Otterdijk, R., \& Meybeck, A. (2011). Global food losses and food waste. Food and agriculture organization of the united nations, Rome.

Hafliðason, T., Ólafsdóttir, G., Bogason, S., \& Stefánsson, G. (2012). Criteria for temperature alerts in cod supply chains. International Journal of Physical Distribution \& Logistics Management, 42(4), 355-371. doi:10.1108/09600031211231335

Heising, J. K., Dekker, M., Bartels, P. V., \& van Boekel, M. A. J. S. (2014). Monitoring the quality of perishable foods: Opportunities for intelligent packaging. Critical Reviews in Food Science and Nutrition, 54(5), $645-654$. doi:10.1080/10408398.2011.600477 PMID:24261537

Hevner, A. R., March, S. T., Park, J., \& Ram, S. (2004). Design Science in Information Systems Research. Management Information Systems Quarterly, 28(1), 75-105. doi:10.2307/25148625

Jedermann, R., Poetsch, T., \& Lang, W. (2014). Smart sensors for the intelligent container. In IEEE 2014 European Conference on Smart Objects, Systems and Technologies (Smart SysTech). IEEE.

Jedermann, R., Ruiz-Garcia, L., \& Lang, W. (2009). Spatial temperature profiling by semi-passive RFID loggers for perishable food transportation. Computers and Electronics in Agriculture, 65(2), 145-154. doi:10.1016/j. compag.2008.08.006

Jevinger, Å., Davidsson, P., \& Persson, J.A. (2011). A framework for agent-based modeling of intelligent goods. In Agents in Principle, Agents in Practice (pp. 97-112). Berlin, Germany: Springer. 
Jevinger, Å., Göransson, M., \& Båth, K. (2014). A field test study on a dynamic shelf life service for perishables. In 26 th Conference of the Nordic Logistics Research Network (NOFOMA).

Jol, S., Kassianenko, A., Wszol, K., \& Oggel, J. (2006). Issues in Time and Temperature Abuse of Refrigerated Foods. Food Safety, 11(6), 30-32.

Larsson, M. (2004). Predicting quality attributes in component-based software systems [Dissertation]. Mälardalen University.

Likar, K., \& Jevšnik, M. (2006). Cold chain maintaining in food trade. Food Control, 17(2), 108-113. doi:10.1016/j.foodcont.2004.09.009

López, T. S., Ranasinghe, D. C., Patkai, B., \& McFarlane, D. (2011). Taxonomy, technology and applications of smart objects. Information Systems Frontiers, 13(2), 281-300. doi:10.1007/s10796-009-9218-4

Mataragas, M., Drosinos, E. H., Vaidanis, A., \& Metaxopoulos, I. (2006). Development of a predictive model for spoilage of cooked cured meat products and its validation under constant and dynamic temperature storage conditions. Journal of Food Science, 71(6), 157-167. doi:10.1111/j.1750-3841.2006.00058.x

Meyer, G. G., Främling, K., \& Holmström, J. (2009). Intelligent products - a survey. Computers in Industry, 60(3), 137-148. doi:10.1016/j.compind.2008.12.005

O'Brien, L., Merson, P., \& Bass, L. (2007). Quality attributes for service-oriented architectures. In International Workshop on Systems Development in SOA Environments. IEEE Computer Society.

Offutt, J. (2002). Quality attributes of web software applications. IEEE Software, 19(2), 25-32. doi: $10.1109 / 52.991329$

Østergaard, N. B., Eklöw, A., \& Dalgaard, P. (2014). Modelling the effect of lactic acid bacteria from starter-and aroma culture on growth of Listeria monocytogenes in cottage cheese. International Journal of Food Microbiology, 188, 15-25. doi:10.1016/j.ijfoodmicro.2014.07.012 PMID:25086348

Peffers, K., Tuunanen, T., Rothenberger, M. A., \& Chatterjee, S. (2007). A design science research methodology for information systems research. Journal of Management Information Systems, 24(3), 45-77. doi:10.2753/ MIS0742-1222240302

Qi, L., Xu, M., Fu, Z., Mira, T., \& Zhang, X. (2014). C 2 SLDS: A WSN-based perishable food shelf-life prediction and LSFO strategy decision support system in cold chain logistics. Food Control, 38, 19-29. doi:10.1016/j. foodcont.2013.09.023

Raab, V., Bruckner, S., Beierle, E., Kampmann, Y., Petersen, B., \& Kreyenschmidt, J. (2008). Generic model for the prediction of remaining shelf life in support of cold chain management in pork and poultry supply chains. Journal on Chain and Network Science, 8(1), 59-73. doi:10.3920/JCNS2008.x089

Ruiz-Garcia, L., Barreiro, P., Rodríguez-Bermejo, J., \& Robla, J. (2007). Review Monitoring the intermodal, refrigerated transport of fruit using sensor networks. Spanish Journal of Agricultural Research, 5(2), 142-156. doi:10.5424/sjar/2007052-234

Saaty, R. W. (1987). The analytic hierarchy process-what it is and how it is used. Mathematical Modelling, 9(3-5), 161-176. doi:10.1016/0270-0255(87)90473-8

Saaty, T. L. (1980). The Analytic Hierarchy Process. New York: McGraw Hill.

Sahin, E., Babaï, M. Z., Dallery, Y., \& Vaillant, R. (2007). Ensuring supply chain safety through time temperature integrators. International Journal of Logistics Management, 18(1), 102-124. doi:10.1108/09574090710748199

Scheer, F. P. (2006). Optimising supply chains using traceability systems. In I. Smith \& A. Furness (Eds.), Improving traceability in food processing and distribution (pp. 52-64). Woodhead Publishing. doi:10.1533/9781845691233.1.52

Sciortino, R., Micale, R., Enea, M., \& La Scalia, G. (2016). A web GIS-based system for real time shelf life prediction. Computers and Electronics in Agriculture, 127, 451-459. doi:10.1016/j.compag.2016.07.004 
Svahnberg, M., Wohlin, C., Lundberg, L., \& Mattsson, M. (2003). A quality-driven decision-support method for identifying software architecture candidates. International Journal of Software Engineering and Knowledge Engineering, 13(05), 547-573. doi:10.1142/S0218194003001421

Verdouw, C. N., Wolfert, J., Beulens, A. J. M., \& Rialland, A. (2016). Virtualization of food supply chains with the internet of things. Journal of Food Engineering, 176, 128-136. doi:10.1016/j.jfoodeng.2015.11.009

Wang, J., \& Yue, H. (2017). Food safety pre-warning system based on data mining for a sustainable food supply chain. Food Control, 73, 223-229. doi:10.1016/j.foodcont.2016.09.048 


\section{APPENDIX 1}

Please provide scores from 1 to 10 , based on importance (10 corresponding to extremely important and 1 not at all important)

Response time: We define the response time as the time it takes from asking for the expiry date, till it is available for the asking user or system. Long response times can be caused by, for instance, poor wireless coverage, overloads, or many and long communication paths. Situations where the expiry date cannot be transmitted at all due to lack of wireless coverage are encompassed by the Availability criteria.

Security: Security corresponds to how difficult it is to manipulate the system into producing no or erroneous expiry dates, or how difficult it is for unauthorized users to access the expiry date. Bad security can be caused by, for instance, communication through networks controlled by unauthorized actors, malicious disturbance of wireless communication and badly protected local information, e.g. in RFID tags.

Availability: Availability is measured as the proportion of total time the service is operational in relation to the required lifetime of the service. We are not focused on implementation-specific details like, for instance, compatibility issues between a reader and a tag or devices of poor quality. Nor do we address the risk of physical damage locally placed devices (for instance RFID tags and sensors) are exposed. Low availability can be caused by, for instance, lack of wireless coverage, overloads, architecture design or batteries running out of power.

Modifiability: Modifiability is a measure of how easy and cost-effectively the software (date estimation algorithms, software functionality etc.) and the hardware components (tags, readers, displays etc.) of the service can be exchanged. Bad modifiability can be caused by, for instance, reusable tags and sensors that may be necessary to update, especially if they lack connection to a central information system.

Scalability: Scalability concerns how well the service system is able to handle a growing number of products or longer transport distances. A solution might have low scalability due to, for instance, local entities responsible for wireless communication or processing from several products, or local entities storing sensor data during long-distance transports.

Accuracy: The accuracy concerns how well the estimated expiry date approximates the actual expiry date. For instance, the closer to the products the sensor is placed, and the more often a sensor measures, the more accurate expiry date can be obtained.

\section{APPENDIX 2}

Table 3. Questionnaire responses

\begin{tabular}{|l|l|l|l|l|l|l|}
\hline & Response time & Security & Availability & Modifiability & Scalability & Accuracy \\
\hline Food producer 1 & 7 & 9 & 8 & 10 & 8 & 10 \\
\hline Food producer 2 & 9 & 10 & 9 & 8 & 8 & 8 \\
\hline Food producer 3 & 7 & 5 & 8 & 8 & 7 & 7 \\
\hline Food producer 3 & 10 & 9 & 10 & 7 & 3 & 10 \\
\hline $\begin{array}{l}\text { Supply chain } \\
\text { manager }\end{array}$ & 5 & 6 & 5 & 3 & 33 & 43 \\
\hline Sum & 38 & 39 & 40 & 36 & 0,144 & 0,188 \\
\hline $\begin{array}{l}\text { Normalized } \\
\text { priorities }\end{array}$ & 0,166 & 0,170 & 0,175 & 0,157 & & 10 \\
\hline
\end{tabular}


Ase Jevinger is a senior lecturer in Computer Science at Malmö University, Sweden. She received her PhD from Malmö University, in 2014. Her research is focused on different applications of the Internet of Things (IoT), mainly within healthcare, logistics and public transports. In particular, she is interested in system architectures, information flow and information needs in loT-based systems. In 2015, Åse Jevinger was awarded "Thesis of the year" (2014) for her PhD thesis, at Malmö University. In addition to her research work, she is the director of research studies in computer science at Malmö University.

Paul Davidsson is Professor of Computer Science at Malmö University, Sweden. He received his Ph.D. in 1996 from Lund University, Sweden. Davidsson is currently the Director of the Internet of Things and People Research Centre. His research interests include agent technology, simulation, information systems, and artificial intelligence. Major application areas are transport systems, building automation, and energy systems. The results of this work have been reported in more than 200 peer-reviewed scientific articles published in international journals, conference proceedings, and books. 